\title{
A mathematical model for the formation of the pricing policy and the plan of the production and transport system in a timber-processing enterprise*
}

\author{
Rodion S. Rogulin \\ E-mail: rafassiaofusa@mail.ru
}

Vladivostok State University of Economics and Service

Address: 41, Gogolya Street, Vladivostok 690014, Russia

Far Eastern Federal University

Address: 10, Ajax Bay, Russky Island, Vladivostok 690922, Russia

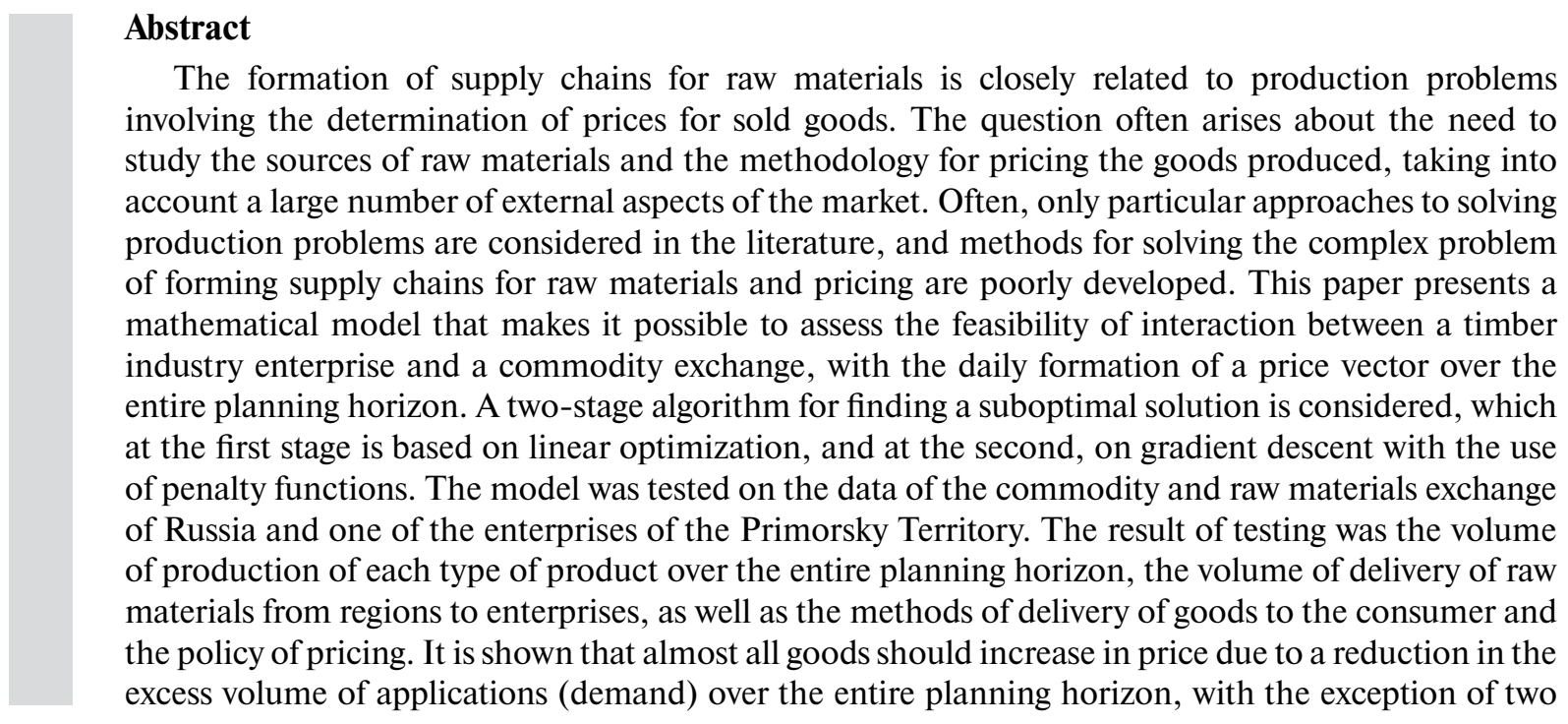

*The article is published with the support of the HSE University Partnership Programme 
types of products. It is noted that the exchange can provide the necessary volume of raw materials for high-capacity production, which demonstrates the possibility, if necessary, to increase the volume of raw materials purchases. It is shown which goods will be included in the release plan more often than others when optimizing the price vector. The ways of delivery of final types of products are analyzed. The disadvantages and advantages of the mathematical model and algorithm are presented.

Key words: price policy; optimization of production; transport task; timber industry; commodity and raw materials exchange; supply chain; output.

Citation: Rogulin R.S. (2021) A mathematical model for the formation of the pricing policy and the plan of the production and transport system in a timber-processing enterprise. Business Informatics, vol. 15, no 3, pp. $60-77$. DOI: $10.17323 / 2587-814 X .2021 .3 .60 .77$

\section{Introduction}

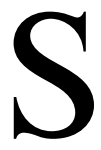
ince the industrial revolution in the 18th and 19th centuries, the importance of optimal production management has increased. Formerly, enterprises, as a rule, produced products and covered only territorially insignificant market volumes with them. This, in turn, required insignificant computational and logical effort, which enterprises could easily cope with. In the aftermath of the Industrial Revolution, businesses began to wonder about expanding markets and increasing production capacity to maximize their profits. However, as enterprises entered new markets, they began to face competition in other regions. When solving the problem of increasing the competitiveness of enterprises, the role of decision-making in relation to entering new markets, determining production volumes and methods of obtaining cheaper raw materials has increased. These three main factors play a significant role in the management of every enterprise today in the 21 st century [1,2].

Among the most acute problems faced by enterprises are the complex issues of production volumes, the method of delivery and the source of raw materials, the method of transporting the final product to the customer, as well as the formation of prices for the final product. The first three problems together represent the task of forming sustainable supply chains. It should be noted that the issues of the formation of supply chains and the pricing policy of the enterprise are not only cornerstone in the enterprise process, but also interrelated and interdependent [1]. It is difficult to solve the above general problem by sequentially solving each of the above four problems separately [2]. The latter problem is especially difficult because it requires an in-depth analysis of markets, production structure and the processing of raw materials [1].

The formation of optimal production plans, in contrast to the formation of the pricing policy of an enterprise, is a problem that has been seriously worked out in world and domestic literature. However, random processes that occur during production make most of these plans inapplicable in practice [2].

The solution to the problem of supply chains has received serious development with the growth of computing power of computers. However, there is no generally accepted approach to solving this problem; only special cases of solving some problems are known [1]. At the same time, the realities of the 21st century force the management of enterprises to think about how to correctly solve this problem exclusively in combination with others, since it is in this combination that the global 
extremum of profit value is achieved. The need for an integrated approach significantly complicates an already very non-trivial problem.

Methods and algorithms for solving transport problems appeared somewhat earlier than methods for solving problems associated with supply chains. However, the problems of traffic flows, equilibria, etc. are still poorly studied and do not give a general understanding of how to solve a particular problem [2].

The formation of a pricing policy for production is a very complex and time-consuming process, since here serious preparatory activities are required for decision-making which often seems unrealizable in a limited time.

In addition, one should not forget about the non-convexity of the admissible set of solutions and the stochasticity of such models, which also gives rise to many problems in the search for solutions [3, 4].

Many parameters of the problem under consideration are probabilistic, which does not allow finding the optimal solution, but there is a possibility of finding a suboptimal solution to the problem. The difference between suboptimal and optimal solutions is that the optimal exists only for the deterministic case, while the suboptimal solution delivers such a value of the objective function that for any probabilistic outcome this value will be acceptable and relatively good [3-5]. However, in the case of a search for a suboptimal solution, there are a number of intractable problems. The nonconvexity of the problem does not allow us to find solutions by generally accepted methods and always requires modification of well-known algorithms and schemes of actions [6].

\section{Literature review}

One of the main issues in the design of the supply chain is the choice of investment method [7]. This perspective in supply chain design requires an understanding of the relationships between costs, supply chain risk factors (including in a global crisis) and investments in supply chain capabilities. The article [7] proposes a multipurpose stochastic model for designing a supply chain under conditions of uncertainty. Risk sources are modeled as a set of scenarios, and the goal is to explore the trade-offs between investing in improving supply chain capabilities and reducing supply chain risks, and minimizing the cost of supply chain disruptions. The results of [7] show that supply chain empowerment can be seen as a mitigation strategy that allows a company to reduce the overall expected value of a supply chain prone to disruptions, including in connection with the COVID-19 pandemic.

The work [8] is devoted to the problem of optimal decision-making in the supply chain, the choice between centralized and decentralized decisions of manufacturers and retailers. The organization and coordination of the supply chain is considered in terms of a carbon tax. Optimization of the supply chain is challenged in three ways: when considering a buyback contract, a subsidy policy, and a joint buyback and subsidy strategy under a carbon tax cap.

In [9], the problem of the supply of raw materials from a commodity exchange to enterprises is considered. The peculiarities of this work are the maintenance of the minimum amount of raw materials in the warehouse, accounting for the supply of raw materials on the exchange, forecasting the supply and prices on the market, drawing the distance traveled by rail with each lot after its purchase. To achieve the goal of the work, a nonlinear stochastic programming model and an algorithm for finding suboptimal solutions were developed.

The study [10] analyzes the management of humanitarian supply chains using the example of India in a normal market situation, not complicated by crises in supply chains during a pandemic. The authors emphasize the importance of information and communication technologies (ICT). The results show that strategic and 
proactive planning is essential to expand the use of ICTs in humanitarian supply chain management. The authors note that this can motivate participants to implement educational programs to raise awareness of the importance of ICTs. These results also support the view that the role of government is critical to expanding the use of ICTs. As argued in [10], an effective, transparent workflow policy associated with the use of a knowledge management system will maximize the benefits of ICT and further improve the efficiency of supply chains.

At the moment, much attention is focused on work in the field of supply chains, where demand is described as a random variable, the value of which can change over time [11, 12]. To take this feature into account, the authors of [13] developed a nonlinear model that allows both parties to a transaction to make decisions that meet all constraints (on both sides) and find the optimal solution in the current situation.

However, it is not always possible to present the problem of the parties as a single mathematical model, and therefore multi-period models come to the fore. Thus, the authors of [14] have developed a two-echelon production and transport distribution network, which is distinguished by the ability to take into account the uncertainty of demand for final products. The purpose of such a model is to determine the minimum values of two parameters at once: the total cost of the supply chain and the time for service. To solve the problem, an L-shaped algorithm was used. The authors of [15] expanded the multi-period threeechelon logistics network of direct and reverse action in conditions of various uncertainties, including demand. The aim of the work was to find such a distribution in which the value of the profit would be maximum. To achieve the goal of the work, echelons of forward and backward distribution of goods were used.

In [16], a two-stage stochastic model was developed to solve the problem of the supply chain on a short-term planning horizon under conditions of uncertainty in demand. The authors were looking for an efficient distribution of goods to consumers based on stochastic demand in order to maximize the final value of the profit. As shown in [17], a special role in problems related to supply chains is played by the simultaneous optimization of heterogeneous and incommensurable parameters. The author's model is multi-product and multi-period; it has a complex stepped architecture that allows for solving the problem of production with random market and organizational demands of buyers and prices for goods.

The stochastic nature of demand very accurately describes the situation of the "sellerbuyer" type and plays an important role in assessing the degree of efficiency of the resulting supply chain.

In [18], a multi-product supply chain was designed to take into account probabilistic scenarios for the development of events. As an algorithm for finding solutions, the authors settled on the branching and binding algorithm. In [19], the problem of placement and distribution of subproblems with random demand was solved in order to be able to make decisions at different levels. The goal of the task was to achieve the maximum profit value. A modified genetic algorithm was used as an algorithm. In the article [20], a new two-purpose mixed-integer problem of supply chain management was formulated which was solved in three classical ways, and the solutions obtained were Pareto-optimal.

Thus, we can assume that the topic under consideration within the framework of the problem of supply chain management is relevant and is rather widely reflected in the literature. However, we note some important features of the above literature review:

- not enough attention is paid to the problems of supply chains and economic policy as a complex problem for any type of production;

- the topic of interaction between timber industry enterprises and commodity exchanges has not been sufficiently worked out. 


\section{Goals, objectives and hypothesis of the research}

Let's consider the activities of enterprises in the timber industry. Three of the most important production processes are involved here: the delivery and volumes of purchases of raw materials from the exchange, the volume of production of each type of product (based on the available stocks of raw materials) and the method of delivery of the final product to the consumer.

For definiteness, it is necessary to note the sources of raw materials supply to the exchange. The exchange enters into agreements with the tenants of the plots from the regions that they can use the platform for trading. After the transaction between the raw material processing enterprise (timber processing complex - the customer) and the cut (the seller), the volume of raw materials declared in the contract ${ }^{1}$ is sent to the customer.

As a rule, enterprises receive requests from customers in advance. In this regard, it seems possible to make the assumption that for planning its activities, an enterprise can use large planning horizons. It should be noted here that the demand for timber products is seasonal, which complicates the planning of the company's activities.

The purpose of the work is to solve the problem of making a decision on the optimal volume of output of goods, on the volumes of purchase of raw materials, on the delivery of finished products to the end consumer and on the formation of the company's pricing policy throughout the entire planning horizon to assess the feasibility of interaction between the enterprise and the timber commodity exchange.

To achieve the goal of the research, the following tasks were set:
1. Construction of an economic and mathematical two-stage model:

a. for the formation of a suboptimal vector of production of products by day on a given horizon for planning the supply chains of raw materials and the vector of volumes of transportation of raw materials to the final consumer;

b. to find an effective vector of prices for the sale of manufactured products.

2. Drawing up an agreed decision for paragraphs (a) and (b) task list;

3. Analysis of model testing results.

We formulate a research hypothesis. It consists of the fact that by increasing the price and, as a consequence, reducing the volume of applications, it is possible to achieve an increase in profits from the sale of timber industry products in the sales markets when interacting with commodity exchanges as sources of raw materials.

\section{Mathematical model}

Any production, including timber industry, is not capable of functioning without a raw material base. To ensure it, it is necessary to determine the plots from where the delivery of raw materials will be carried out. For this we will use the services of the St. Petersburg International Commodity Exchange (SPIMEX ${ }^{2}$ ). Every day, the exchange publishes data on how many transactions (orders) were made, at what price and what volume of raw materials was sold. In addition, the exchange provides services for the delivery of raw materials to the consumer, which is also included in the price of the goods. Many regions are represented on the exchange where raw materials can potentially come from.

\footnotetext{
${ }^{1}$ The purchase and sale agreement specifies the methods and price of timber delivery.

Delivery can be carried out by the enterprise, however, further we consider the delivery of raw materials by the supplier

${ }^{2}$ https://spimex.com/
} 
After the receipt of a sufficient volume of raw materials at the production warehouse, the enterprise must decide on the optimal vector for the production of the final product, with a focus on demand. Once the production plans have been implemented, the goods must be delivered to customers in accordance with their demand, using known transport hubs.

To solve the problem, we introduce the following variables and designations [21]:

$k$-type (number) of production, $k=1, \ldots, K$;

$l$-type (number) of raw material for production, $l=1, \ldots, L$;

$m$ - day number within the considered planning horizon, $m=1, \ldots, M$;

$w$ - week number within the considered planning horizon (determined depending on the day number), $w=1, \ldots, W$;

$s$ - month number within the considered planning horizon, $s=1, \ldots, S$;

$c_{i l r m}-$ buying price of $i$ lot with type raw material type $l$ in region $r$ during the day $m$ (rub.), including delivery costs;

$V_{i l r m}$ - type raw material volume in lot from region $r$ during the $m$ day $\left(\mathrm{m}^{3}\right)$;

$v_{i l r m}$ - raw material bought volume of $l$ raw material in lot $i$ from region during day $m\left(\mathrm{~m}^{3}\right)$;

$u_{l m}$ - stock in the warehouse of raw materials type $l$ during day $m\left(\mathrm{~m}^{3}\right)$;

$u_{m}^{\max }-$ maximal capacity rate of warehouse during day $m\left(\mathrm{~m}^{3}\right)$;

$u_{m}^{\text {min }}$ - the level of an inviolable (safety) stock of raw materials of type $l$ in the warehouse on any day over the entire planning horizon $\left(\mathrm{m}^{3}\right)$;

$v_{i l r}^{\prime}$ - volume of raw materials of type $l$ purchased in the previous period about which it is known that it will arrive at the warehouse on the day $m\left(\mathrm{~m}^{3}\right)$;

$A_{l k}^{s}$ - the resource costs volume $l$ for the unit production of output $k$ in a month $s\left(\mathrm{~m}^{3}\right)$;

$p_{k m}-$ selling price $k$ of product type $l$ in day $m$ (rub.);
$N$ - number of independent simulation iterations;

$F C_{n}$ - fixed cost for simulated iteration $n$ (rub.), $n=1, \ldots, N$;

$x_{k m}$ - volume of production of goods type $k$ during day $m$ (pcs.);

$z_{i j k w}$ - volume of products $k$ transported between points $i$ and $j$ during week $w$ (pcs.);

$Z_{i j w}$ - the throughput of the arc between points $i$ and $j$ in a week $w$ (pcs.);

$\tilde{z}_{i j w}-$ road use indicator from point $i$ to point $j$ in week $w$ in the transport subproblem solution graph;

$c_{i j w}-$ transportation costs from point $i$ to point $j$ in week $w$ (rub.);

$I$ - the number of orders that were purchased by the enterprise in the previous period (until the moment $m=0$ ) and for which the dates of arrival at the warehouse are known;

$R$ - the number of regions from which applications are received;

$T_{r}$ - the rate of time spent (in days) for the delivery of any volume of raw materials from region $r$ by rail, $r=1, \ldots, R$;

$Q_{j^{*} k w}-$ demand of retailer $j^{*}$ for products $k$ in week $w$;

$\bar{Q}_{j^{\prime k w}}$ - the demand volume expectation of a retail company $j^{*}$ for products $k$ per week $w$;

$M_{1}$ - the number of days of the current period for which purchased bids will arrive at the warehouse during the next period;

$J$ - set of vertices in a transport graph;

$J^{\text {out }}-$ set of vertices incident to a production point;

$J^{*}-$ many retailers (final destination of manufactured products);

$B_{0}$ - the company's budget allocated for trading as of the moment the model starts working;

$x_{k m 1}-$ hypothetical production volume of product type $k$ in day $m=M+1, \ldots, M_{1}$, which is defined as follows: 


$$
\begin{aligned}
\dot{x}_{k m 1} & =\left\lceil\frac { 1 } { 2 } \left(\max _{m^{*} \in[m-t, m]}\left(x_{k m^{*}}, x_{k\left(m^{*}-1\right) 1}\right)+,\right.\right. \\
& \left.\left.+\min _{m^{*} \in[m-t, m]}\left(x_{k m^{*}}, \dot{x}_{k\left(m^{*}-1\right) 1}\right)\right)\right]
\end{aligned}
$$

where $t$ is the number of days for which the maximum and minimum values of are selected in the constraints below.

The values of the parameters $u_{l 0}, B_{0}$ are set and correspond to the last day of the previous period.

As an objective function, we will consider the pre-tax profit of the enterprise on the planning horizon $M$. Then the mathematical notation of the optimization problem takes the following form:

$$
\begin{gathered}
\max _{p} \max _{x, v, z, z} \sum_{m}\left(\sum_{k} p_{k m} x_{k m}-\sum_{i, l, r} c_{i l r m} v_{i l r m}-\sum_{i, j} c_{i j w} \tilde{z}_{i j w}\right) \\
u_{l m}=u_{l(m-1)}+\sum_{i, r} v_{i l r\left(m-T_{r}\right)}+\sum_{i, r} v_{i l r\left(m-T_{r}\right)}^{\prime}-\sum_{k} A_{l k}^{s} x_{k m} \\
l=1, \ldots, L ; m=1, \ldots, M
\end{gathered}
$$$$
\sum_{l} u_{l m} \leq u_{m}^{\max } ; m=1, \ldots, M+M_{1}
$$$$
u_{l m} \geq u_{l}^{\min } ; l=1, \ldots, L ; m=1, \ldots, M+M_{1}
$$$$
u_{l m} \geq u_{l}^{\min } ; l=1, \ldots, L ; m=1, \ldots, M+M_{1}
$$$$
B_{0}+\sum_{m=1}^{m^{*}}\left(\sum_{k} p_{k m} x_{k m}-\sum_{i, l, r} c_{i l r m} v_{i l r m}-\right.
$$$$
\left.-\sum_{i, j} c_{i j w} \tilde{z}_{i j w}-F C_{n}\right) \geq 0
$$$$
m^{*}=1, \ldots, M
$$$$
\sum_{j} z_{i j k w}=\sum_{i} z_{i j k w}
$$$$
k=1, \ldots, K ; w=1, \ldots, W ; i, j \in J
$$$$
\sum_{j \in J^{\text {out }}} z_{1 j k w}=\sum_{m=(w-1) \cdot 7+1}^{w 7} x_{k m} ; k=1, \ldots, K ; w=1, \ldots, W
$$

$$
\begin{aligned}
& \sum_{i} z_{i j^{*} k w} \leq Q_{j^{*} k w} ; \\
& j^{*} \in J^{*} ; k=1, \ldots, K ; w=1, \ldots, W \\
& \sum_{k} z_{i j k w} \leq \tilde{z}_{i j w} Z_{i j w} ; i, j \in J ; w=1, \ldots, W \\
& B_{0}=\text { const } \\
& u_{10}=\text { const } \\
& u_{l m}, x_{k m}, v_{i l r m}, \in \mathrm{Z}^{+} \\
& z_{i j k w} \geq 0 \\
& \tilde{z}_{i j w} \in\{0 ; 1\} \\
& u_{l m}=u_{l(m-1)}+\sum_{i r} v_{i l r\left(m-T_{r}\right)}+\sum_{i r} v_{i l r\left(m-T_{r}\right)}^{\prime}- \\
& -\sum_{k} A_{l k}^{s} x_{k m 1} ; l=1, \ldots, L ; m=M+1, \ldots, M_{1} \\
& p_{k m}=\mathrm{const} ; m=1, \ldots, 7 ; k=1, \ldots, K \\
& \left|\frac{p_{k(m+1)}-p_{k m}}{p_{k m}}\right| \leq \gamma ; m>7 ; k=1, \ldots, K \\
& A_{l k}^{s+1}=\max \left(0, \min \left(A_{l k}^{s}, A_{l k}^{s}+\varepsilon_{l k}^{(1)}\right)\right) ; \\
& \varepsilon_{l k}^{(1)} \in\left[-\alpha^{(1)}, \beta^{(1)}\right] \\
& u_{m+1}^{\max }=\max \left(u_{m}^{\max }, u_{m}^{\max }+\varepsilon_{m}^{(2)}\right), \\
& \varepsilon_{m}^{(2)} \in\left[-\alpha^{(2)}, \beta^{(2)}\right] \\
& Q_{n k w}=\left(\bar{Q}_{n k w}+\varepsilon_{n k w}^{(4)}\right) \cdot \rho_{n k w} ; \varepsilon_{n k w}^{(4)} \in\left[-\alpha^{(4)}, \beta^{(4)}\right] \\
& \int \frac{c^{(2)}}{\left(1+\sum_{m=(w-1) \cdot 7+1}^{w \cdot 7} \frac{\left(p_{k(m+7)}-p_{k m}\right)}{7 \cdot p_{k m}}\right)^{c^{(1)}}},
\end{aligned}
$$

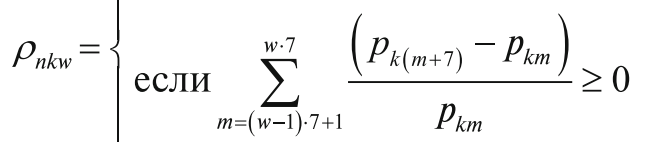

$$
\begin{aligned}
& \left.c^{(2)}\left(1+\mid \sum_{m=(w-1) \cdot 7+1}^{w \cdot 7} \frac{\left(p_{k(m+7)}-p_{k m}\right)}{7 \cdot p_{k m}}\right)\right)^{c^{(1)}} \text {, иначе }
\end{aligned}
$$




$$
\begin{aligned}
& F C_{n} \in\left[\alpha^{(3)}, \beta^{(3)}\right] \\
& Z_{i j w} \in\left[\alpha^{(5)}, \beta^{(5)}\right] \\
& c_{i j w} \in\left[\alpha^{(6)}, \beta^{(6)}\right]
\end{aligned}
$$

where $\varepsilon_{l k}^{(1)}, \varepsilon_{m}^{(2)}, F C_{n}, Z_{i j w}, c_{i j w}$ - uniformly distributed random variables ${ }^{3}$;

$\varepsilon_{j^{* k k w}}^{(4)}-$ noise component ${ }^{4}$ of random value $\bar{Q}_{n k w}{ }^{j k w}$ at the starting week $w$ for the product $k$ for retailer $j^{*}$;

$$
\begin{gathered}
\alpha^{g}, \beta^{g} \geq 0 ; g=1, \ldots, 6 ; \\
\alpha^{g}, \beta^{g} \in \mathrm{Z}^{+} ; g=1, \ldots, 3 .
\end{gathered}
$$

Let us consider expressions (1-26) in more detail. The goal of problem (1) is to find such a combinatorial distribution $p, x, v, z, \tilde{z}$, that the value of profit is maximized. The volume of raw materials in the warehouse is monitored every day (2). Any warehouse has its maximum capacity (3), and the volume of incoming raw materials cannot exceed it. Since any production is associated with a certain risk that raw materials can reach the warehouse a little later than expected, we introduce the concept of an inviolable (safety) stock of raw materials of each type (4). Constraint (5) states that the volume of production is limited by the amount of raw materials available in the warehouse. From constraint (6) it follows that the amount of accumulated profit for each day of production cannot take a negative value. Constraint (7) says that from each request it is impossible to buy more raw materials than stated. The volume of the input flow of the finished product is equal to the output flow (8), in other words, no goods can remain at the intermediate points of transportation of the finished product. The volume of finished products that was produced in a working week is fed to the graph (9). The volume of the incoming flow of finished pro- ducts to the vertices with the consumer cannot exceed his volume of demand (10). Constraint (11) allows you to track the use of different graph arcs along which the finished product is transported. Formulas (12) and (13) assert that the initial budget of the company and the volume of raw materials in the warehouse are initially given. Formulas (14-16) reflect the nature of the optimization variables. Constraint (17) makes it possible to continue the formation of the vector of purchases of raw materials, the vector of production, the vector of the method of delivery of goods, taking into account a small additional period, if the model will be used for several periods. Formula (18) reflects the fact that prices are fixed on the first day of operation of the enterprise. Formula (19) shows that the price can change by no more than $100 \cdot \gamma \%$. Formulas (20-26) reflect the stochastic nature of the changes, in other words, for each realization of such random variables, the optimal or suboptimal solution of the system will be determined.

As follows from formulas (1-26), the model is nonlinear and stochastic. The resulting problem, according to [22], can be classified as a stochastic programming problem with a deterministic objective function and stochastic constraints. The search for the optimal solution in stochastic programming problems is nontrivial and always requires a special approach, especially if the objective function or constraints are nonlinear [22].

Consider approaches to solving the problem. The most widely known methods are the method of deterministic equivalent (MDE), the confidence method (DM) (better known as the generalized minimax approach [22-24]) and some others. MDE allows you to move away from stochastic constraints to constraints of a deterministic nature and to reduce the original stochastic programming problem to the problem of "deter-

\footnotetext{
${ }^{3}$ Since the nature of these quantities is unknown, it is assumed that they are evenly distributed

${ }^{4}$ Noise is usually understood to mean some random deviation
} 
ministic" optimization, which allows you to expand the toolkit for solving the problem. However, this method cannot always be applied, since the main statements inherent in this method impose rather stringent requirements on the structure of the loss function and the distribution of the vector of random parameters. In some cases, it is possible to successfully estimate the real value of the quantile function using some upper bound of the confidence level. Since such an estimate is a maximum function on the corresponding confidence set, the problem of minimizing the quantile function can be replaced by a minimax problem, the solution of which can be obtained numerically or analytically $[22,25]$.

In [26], a statement of a two-stage linear stochastic programming problem was proposed. The novelty of the statement in this article is that the constraints of the second stage problem are a linear complementarity problem with a deterministic compensation matrix. The introduction of such constraints allows one to obtain compensation for the design at the "solvability limit" which is provided by the conditions of complementary slackness of the complementarity problem.

Relying on a brief review of existing works, we note that problem $(1-26)$ can be reduced to a two-stage model, where at the first step the values of stochastic parameters are played out, the price vector is fixed, and the classical mixedinteger programming problem (1-16) is solved, and in the second step, penalty functions and the gradient descent method are applied to change the vector of sales prices for goods.

To solve problem (1-26), we will conduct an iterative search for a suboptimal solution using two subtasks - stages (search for an optimal production plan, delivery of raw materials and goods, and search for a suboptimal price vector for selling goods).
Stage 1. Solve problem (2-16) with an objective function of the form (27):

$$
\max _{x, v, z, z} \sum_{m}\left(\sum_{k} p_{k m} x_{k m}-\sum_{i, l, r} c_{i l r m} v_{i l r m}-\sum_{i, j} c_{i j w} \tilde{z}_{i j w}\right),
$$

where $\quad x=\left\{x_{k m}\right\}_{k m}, v=\left\{v_{i l r m}\right\}_{i l r m}, z=\left\{z_{i j k w}\right\}_{i j k w}$, $\tilde{z}=\left\{\tilde{z}_{i j w}\right\}_{i j w}$.

At the first iteration, the vector is generated randomly with a given initial condition (18). The demand value is calculated using (18, 19,23 ) (if you reapply stage 1 , the next iteration will use the price values obtained in the previous iteration of stage 2 ). The values from expressions (20-26) are played every iteration. The search for a solution to the problem is carried out using the Gomori algorithm included in the system MathWorks 5 .

Stage 2. For fixed values $x_{k m}, v_{i l r m}, z_{i j k w}, \tilde{z}_{i j w}$, obtained in stage 1 , we are to solve the optimization problem $(10,22,23,28,29)$ :

$$
\begin{gathered}
\max _{p} \sum_{m}\left(\sum_{k} p_{k m} x_{k m}-\sum_{i, l, r} c_{i l r m} v_{i l r m}-\sum_{i, j} c_{i j w} \tilde{z}_{i j w}\right) \\
p_{k m^{*}} \cdot(1-\gamma) \leq p_{k 1+m^{*}} \leq p_{k m^{*}} \cdot(1+\gamma) \\
m^{*}=1, \ldots, M-1
\end{gathered}
$$

Stage 3. After stage 2, it is necessary to check the value of the difference in profit values. Repeat successively stages 1 and 2 until the value of profit $g$ of iterations in a row begins to change by less than the value of $\wp$, exit the algorithm.

As you can see, at stage 1, linear optimization is carried out: the problem of mixed-integer linear programming is solved. However, the second stage is optimization of the nonlinear functional. To find the optimal solution, we use gradient descent $[27,28]$ and penalty functions [29-31]. Then problem $(10,18,22,23,28,29)$ takes the form $(18,22,23,30-34,36)$ with the iteration rule:

\footnotetext{
${ }^{5}$ MathWorks. Documentation. Mixed-Integer Linear Programming Algorithms: https://

it.mathworks.com/help/optim/ug/mixed-integer-linear-programming-algorithms.html
} 


$$
\begin{aligned}
& F(p)=\sum_{m}\left(\sum_{k} p_{k m} x_{k m}-\sum_{i, l, r} c_{i l r m} v_{i l r m}\right. \\
& \left.-\sum_{, i j} c_{i j w} \tilde{z}_{i j w}\right)+\frac{\lambda^{1}}{2}\left[G_{1}\right]_{-}^{2}-\lambda^{2} G_{2} \rightarrow \max _{p} \\
& G_{1}\left(p, j^{*}, k, m\right)=\sum_{i} z_{i j^{*} k w}- \\
& -\left(\bar{Q}_{n k w}+\varepsilon_{j^{*} k w}^{(4)}\right) \cdot \rho_{n k w}(p) \\
& G_{2}(p, k, m)=\frac{\left(p_{k 1+m}-p_{k m}\right)_{+}^{2}}{2}+ \\
& +\frac{\left(p_{k m}-p_{k 1+m}\right)_{+}^{2}}{2} \geq 0 \\
& \left(p_{k 1+m}-p_{k m}\right)_{+}^{2}=\left\{\begin{array}{l}
\left(p_{k 1+m}-p_{k m}\right)^{2}, \\
\text { если } p_{k 1+m}-p_{k m} \cdot(1-\gamma) \leq 0 \\
0, \text { иначе }
\end{array}\right. \\
& \left(p_{k m}-p_{k 1+m}\right)_{+}^{2}=\left\{\begin{array}{l}
\left(p_{k m}-p_{k 1+m}\right)^{2}, \\
\text { если } p_{k m} \cdot(1+\gamma)-p_{k 1+m} \leq 0 \\
0, \text { иначе }
\end{array}\right. \\
& p^{(k+1)}=p^{(k)}-h \nabla F\left(p^{(k)}\right) \\
& {\left[G_{1}\left(p, j^{*}, k, m\right)\right]_{-}^{2}=\min \left(0,\left(\sum_{i} z_{i j^{*} k w}-\right.\right.} \\
& \left.\left.-\left(\bar{Q}_{n k w}+\varepsilon_{j{ }^{* k w}}^{(4)}\right) \cdot \rho_{n k w}(p)\right)\right)^{2}
\end{aligned}
$$

where $m=1, \ldots, M$;

$p^{(k)}-$ price vector on iteration $k$;

$h$-step value;

$\lambda^{1}=\left(\lambda_{j^{*} k m}^{1}\right)_{j^{*} k m}$ and $\lambda^{2}=\left(\lambda_{k m}^{2}\right)_{k m}-$ vectors, which elements are big enough numbers.

The stopping criterion will be considered the achievement of such a value of $k$ so that the inequality

$$
\nabla F\left(p^{(k+1)}\right)-\nabla F\left(p^{(k)}\right) \leq \wp
$$

Let us consider formulas (30-35) in more detail. Formula (30) - objective function with penalties

$$
\frac{\lambda^{1}}{2}\left[G_{1}\right]_{-}^{2}, \lambda^{2} G_{2}
$$

Constraint (31) reflects the difference between the volume of products delivered to the customer and the value of the demand of the same customer. If the value of the price goes beyond the limits $(32-34,36)$, then the objective function receives a penalty. Formula (35) is a rule for calculating the price vector.

The solution will tend to a state where prices will meet the constraints, and the volume of orders for goods will tend to the volume of products delivered. The latter will allow us to increase the price to the maximum and reduce the number of applications and costs in the production and delivery of finished goods, which will also increase the value of profit to a suboptimal value.

\section{Testing the model}

One of the leading timber industry enterprises of the Primorsky Territory was chosen to test the model. The company seeks to extract maximum profit from its activities and work in compliance with the current legislation, therefore the company is considering the possibility of cooperation only with the exchange. Since the company is only trying to assess the feasibility of cooperation with the exchange, we will use the already published data of the exchange for the entire period. However, we will focus on the interval from $02 / 01 / 2020$ to $11 / 31 / 2020$, because there is a seasonal decline in the demand for goods and supply in the raw materials market. During the period under review, 1509 deals (orders) were published. Enterprises from four regions are represented to the greatest extent on the exchange: Irkutsk Region $(r=1)$, Republic of Udmurtia $(r=2)$, Moscow Region $(r=3)$ and Perm Territory $(r=4)$. Knowing the coordinates of the enterprises, it would be possible to conduct a dialogue with them directly, bypassing the exchange. However, the exchange hides the real coordinates, so all transactions are carried out through the exchange both from the buyer's side and from the supplier's side. 
An array of the following data was collected from the official website of the exchange for the specified period: prices of offered orders $c_{i r m}$, volumes of orders $v_{i l r m}$, selling prices of final goods $p_{k 1}$, the number of orders for each type of raw material. In addition, based on the analysis of the company's sales statistics, we will assume that the daily demand $\sum Q_{n k m}{ }^{6}$ for each type of product is set on the considered planning horizon.

To solve the transport block and compile the adjacency matrix of transport intermediate and final points, we will use the map of international transport corridors of Eurasian railways ${ }^{7}$. To estimate the throughput, we will use the data of the enterprise.

The main initial data characterizing the enterprise are presented in tables $1-3$.

Let us consider the requirements for the computing power of the computer with the help of which we have to find a solution to the problem, and note some features.

The number of constraints and variables was 10189 and 49779, respectively. This allows us to assert that the constraint matrix is weakly sparse, which, in turn, increases the search time for a solution. In view of this, the maximum number of vertices of the polyhedral feasible set of solutions was limited to 1500 units, which is extremely small (but even for such a number of vertices, the search for solutions will be very long).

To speed up the search for a solution, we will use a 12-thread Ryzon 2600x processor and 64 GB RAM, as well as a parallel programming technique. The search for a solution for each problem in stage 1 will be computed in parallel, and this is exactly what requires a large amount of RAM. Let us clarify that such an amount of RAM is required only at the step of forming con-
Table 1.

\section{The main initial parameters of the enterprise}

\begin{tabular}{c|c}
\hline Parameters & Values \\
\hline$u^{\text {max }} \cdot \mathrm{m}^{3}$ & 7750 \\
\hline$u_{l}^{\text {min }}, \forall l . \mathrm{m}^{3}$ & 50 \\
\hline$u_{l 0} \cdot \mathrm{m}^{3}$ & $(2250 ; 2250)$ \\
\hline $\begin{array}{c}p_{k 1} \cdot k=1, \ldots, 9, \\
\text { thousand rubles }\end{array}$ & $\begin{array}{c}35 ; 52.89 ; 73.4 ; 83.54 ; 87.125 ; \\
97.47 ; 11.72 ; 121.97 ; 143.4)\end{array}$ \\
\hline$T_{r} \cdot$ days & $(3 ; 5 ; 6 ; 5)$ \\
\hline$B_{0} \cdot$ rubles & 20000000 \\
\hline$h$ & $\max (150 ; 1000-25 k)$ \\
\hline$\wp$ & $1 \mathrm{e}-02$ \\
\hline$g$ & 2 \\
\hline$\lambda_{j^{*} k m}^{1}$ & $1 \mathrm{e}+04$ \\
\hline$\lambda_{k m}^{2}$ & $1 \mathrm{e}+04$ \\
\hline$c^{1}$ & 0.5 \\
\hline$c^{2}$ & 1 \\
\hline$\gamma$ & 0.006 \\
\hline
\end{tabular}

straint matrices and objective functions for different iterations.

For calculations, we will use the high-level programming language Matlab and a function from the intlinprog ${ }^{8}$ extension package to find solutions to linear optimization problems. In order to speed up the process of finding solutions, we deliberately did not impose an integer constraint on the $z$ variables. This will not affect the correctness of the solution of the problem, because in

\footnotetext{
${ }^{6}$ For future research in the field of modeling production processes from the field of supply chains, it is planned to predict the demand for the manufacturer's products based on the data available to the enterprise. As for this work, here the volume of demand for 2020, noisy by the author, was used and the values of prices for the sale of final goods were estimated

${ }^{7}$ http://www.expresstk.ru/wp-content/uploads/2017/08/Evroaziatskie-transportnye-koridory.pdf

${ }^{8}$ https://www.mathworks.com/help/optim/ug/intlinprog.html
} 
The cost of raw materials to produce a unit of production

Table 2.

\begin{tabular}{c|c|c|c|c|c|c|c|c|c|c}
\hline Raw material $(l) \backslash$ product no $(k)$ & $\mathbf{1}$ & $\mathbf{2}$ & $\mathbf{3}$ & $\mathbf{4}$ & $\mathbf{5}$ & $\mathbf{6}$ & $\mathbf{7}$ & $\mathbf{8}$ & $\mathbf{9}$ & $\sum_{k} A_{l k}$ \\
\hline 1 & 3 & 4 & 5 & 4 & 5 & 5 & 7 & 5 & 9 & $\mathbf{4 7}$ \\
\hline 2 & 2 & 4 & 4 & 6 & 6 & 7 & 6 & 9 & 7 & $\mathbf{5 1}$ \\
\hline
\end{tabular}

Table 3.

Ranges of values of some random variables

\begin{tabular}{c|c|c|c}
\hline Parameters & Values & Parameters & Values \\
\hline$\alpha^{(1)}$ & 0.1 & $\beta^{(1)}$ & 10 \\
\hline$\alpha^{(2)}$ & 100 & $\beta^{(2)}$ & 100 \\
\hline$\alpha^{(3)}$ & 2000000 & $\beta^{(3)}$ & 2820000 \\
\hline
\end{tabular}

the theory of the transport problem, it is proved that the solution of such a subproblem is always integer [21].

\section{Discussion}

Consider the behavior of profit over the entire planning horizon (Figure 1). As you can see from the figure, the profit value changed nonmonotonously. This is because at each step random variables were played $(20,21,24-26)$ and $\varepsilon_{j^{*} k w}^{(4)}-$ products demand noise component. The last element especially strongly influenced the final value of the volume of demand for the products, which, in turn, was reflected in the profit indicator.

The last profit value (after the stop criterion was triggered) was played four times to understand the approximate spread of this value depending on the implementations of the random variables.

When testing the final solution, we managed to notice the following. The behavior of accumulated profit stabilizes in three out of four cases (this can be observed especially well when analyzing the behavior of profit by day). From the decision-making point of view, it is very difficult to find a solution on the interval between the first and eightieth days of the planning horizon. The difficulty lies in choosing such values of the vectors $p, x, v, z, \tilde{z}$, so that the profit values are positive.

Note that, for commercial reasons, we cannot reflect the demand $\dot{Q}_{j^{*} k w}$, however, it can be noted that the demand has a decreasing trend towards summer, and after summer - an upward trend.

We also note that the hypothesis put forward about an increase in the value of profit due to an increase in price and, as a result, a decrease in the volume of incoming orders for goods when interacting with a commodity exchange as a source of raw materials for production, is confirmed.

A similar trend can be seen with the supply of raw materials of types 1 and 2 on the exchange.

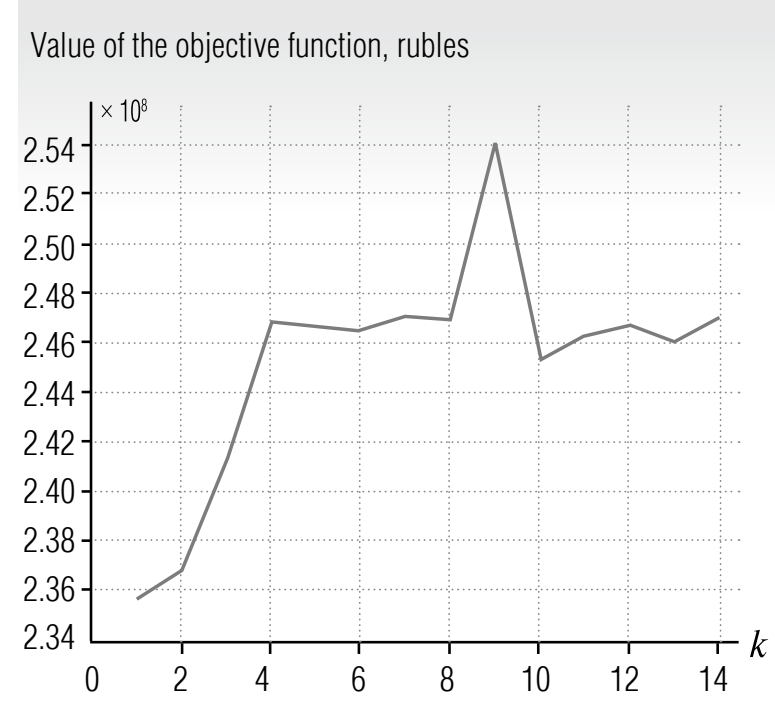

Fig. 1. Visualization of the change in the profit indicator depending on the price value $p^{(k)}$ at iteration $k$ 
This is because in summer many places for the extraction of raw materials are difficult to access due to swampiness and hard passability [21]. However, the values of the supply of raw materials do not differ greatly by months, which is associated with the violation of legislation in the field of forest use [21]. It can be concluded that the volume of purchases on the exchange is insignificant relative to the supply, which, in turn, does not lead to a sharp rise in prices and a shortage of raw materials for production. In other words, the exchange can fully provide the enterprise with all the necessary types and volumes of raw materials without any special additional load.

The fall in demand for goods and supply of raw materials leads to a complex management situation in production - there is no clear understanding of how to act in such a way that the condition of a positive value of the accumulated profit for each day during the entire planning horizon is fulfilled. If this condition is not met, then it is necessary to raise the question of attracting investment, which significantly complicates the original problem.

Consider the volume of purchases of raw materials by region. Despite the transport proximity of the Irkutsk Region to the Primorsky Territory, where the enterprise is located, and despite the maximum volume of supply on the exchange of raw materials from the Irkutsk Region, preference was given to purchases from the Republic of Udmurtia $(\approx 40 \%)$ and the Moscow Region $(\approx 30 \%)$. This is very different from the procurement results shown in [21], where the Irkutsk region dominates in the supply of raw materials. This is because in the current work the factors of price changes for the final product are additionally considered, the transport subproblem is solved, and the possibility of choosing the volume of raw materials from the order from the exchange 9 is provided. The influence of these three factors on the change in the volume of purchases is supposed to be considered in subsequent works.

Consider the volume of production of a particular type of product. Type 9 is the most popular product. In addition, for this product, one can observe a difference from the general trend: the closer to summer, the higher the demand. Probably, this can be explained by the fact that the price for this product is the highest, and the ratio of costs to revenue is the smallest in comparison with similar indicators of other types of products.

As for product 8, its production increases from February to April and falls after April.

The complex dynamics of changes in production volumes is demonstrated by product 7 . Until September, it is difficult to speak about the nature of changes in production volumes of this product, but it is safe to say that this type of product should be produced in significant volumes in September and October, after the seasonal decline in demand comes out.

It can be argued with a high degree of certainty that the feasibility of its production of commodity 6 is extremely small.

Moreover, the dynamics of changes in production volumes, which are difficult to analyze, are demonstrated by goods 1-5. Even though they are often produced, their volumes are insignificant against the general background of the production of other goods.

Consider the situation that occurred in the warehouse during production. Let us introduce the operator ave $(X)$, the result of which is the average value of $X$ at all iterations. Here you can see that the amplitude of the stocks of raw materials in the warehouse is significant for each iteration. This allows us to assert that the degree of complexity of the production planning process significantly depends on the procedure for replenishing the warehouse with raw materials. It is worth noting here that, on average, the

\footnotetext{
9 Work [19] shows another method in play on the exchange, when a bid can be bought out only entirely
} 
results of the model show that the warehouse occupancy rate is above $50 \%$. This is because in summer the cost of raw materials increases, and closer to cold periods (winter, late autumn and early spring) it decreases. The simulation results show that in order not to buy raw materials in the summer at an overpriced price, they should stock up a little earlier.

In mid-April and June, one can observe that, on average, the stock of raw materials in the warehouse reaches its peak $u_{m}^{\max }$, and that almost every day (97\%) throughout the planning horizon, there is a purchase and receipt of raw materials at the warehouse, which together also complicates the process inventory planning.

Consider the transport sub-problem (Figure 2). Here, those routes are marked in black, along which the ratio of the number of transported goods to the throughput of the arc exceeds $66.67 \%$. For routes indicated by gray and dotted lines, these ratios are above $33.33 \%$ and below $66.67 \%$.

The main routes go through points $1 \rightarrow 2 \rightarrow 6$ to point 10 , as well as from point 1 to point 8 :
$1 \rightarrow 3 \rightarrow 4 \rightarrow 8$. For the case with vertex 9 , the situation is somewhat different. Here two streams merge into one: this is part of the stream $1 \rightarrow 2 \rightarrow 6 \rightarrow 5 \rightarrow 9$ and $1 \rightarrow 3 \rightarrow 4 \rightarrow 5 \rightarrow 9$, where on average the second stream accounts for $65.71 \%$ of the cargo turnover for the top 9 .

However, there are other routes that are less frequently used $(2 \rightarrow 3,3 \rightarrow 2,6 \rightarrow 7,5 \rightarrow 6$, $5 \rightarrow 4)$. These routes are used very rarely; their average occupancy rate does not exceed $0.56 \%$. This is mainly because these routes are not on the way to the final customer. Note that there are routes that are more significant in terms of occupancy. Among them, it is worth considering $6 \rightarrow 5,5 \rightarrow 7,7 \rightarrow 10$. Here, graph arcs are used because the main routes cannot cope with the flow of goods.

The main task of this study is to identify how the price for each type of product will change based on the calculation so that when solving problem $(1-16)$, the final value of profit is maximum. To do this, consider the drawings from the online application ${ }^{10}$.

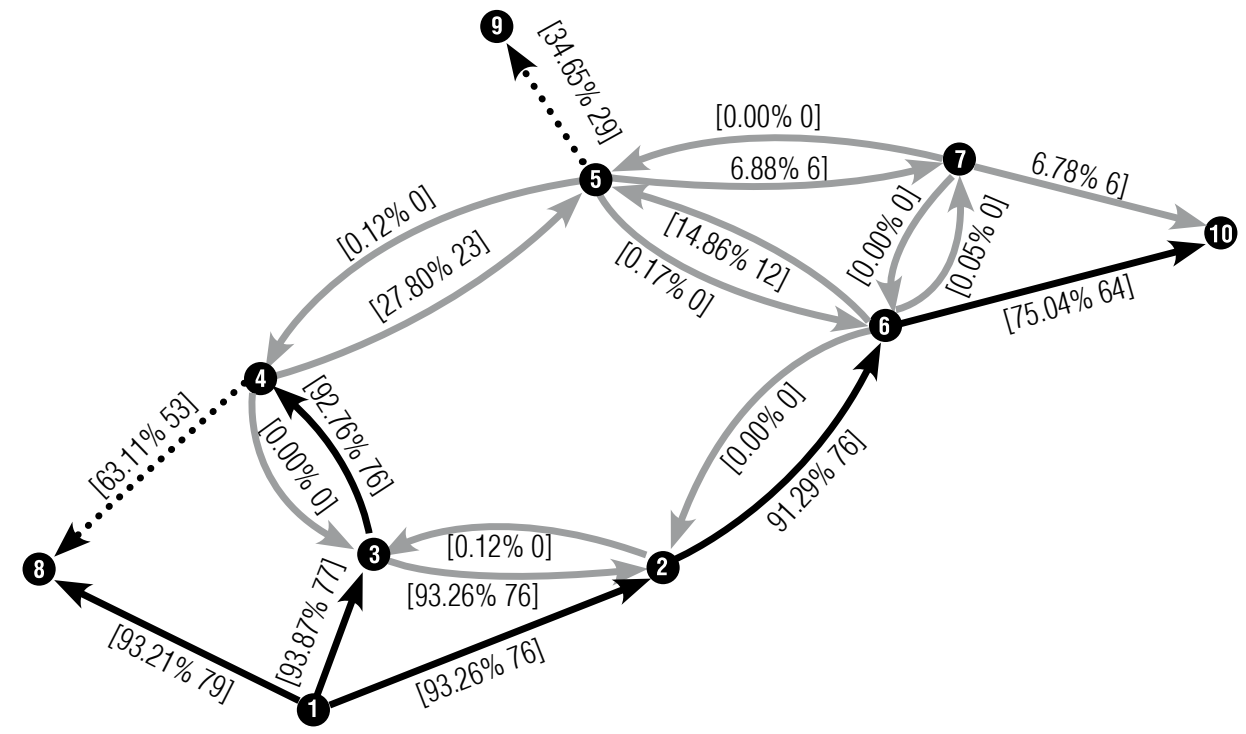

Fig. 2. Visualization of the graph of railway routes with notes about the average degree of "fullness" of the arcs over the entire planning horizon

\footnotetext{
${ }^{10}$ https://drive.google.com/drive/folders/1DM2YsxBHPOZLvaTHjbmP8GK3cVa DfDa5? usp=sharing
} 
For goods of any type, except for the first and ninth, a stable increase in price is characteristic, which in the context of $(22,23)$ means that due to an increase in price, a decrease in the excess volume of demand for goods is achieved and, as a consequence, the value of the final profit increases. For goods of the first and ninth types, the situation is somewhat different. For the first type of product, the volume of demand decreases throughout the summer period due to an obvious increase in prices, and for the ninth type, a price increase is observed, which stabilizes as the winter period approaches. The nature of these observations out of the rule is expected to be studied further.

Let's consider the positive and negative aspects of the model and the two-stage optimization scheme.

The positive aspects of the model include the relative conceptual simplicity of the study in terms of modeling and optimization stages. All algorithms and methods used at these stages are well researched. Many modifications of their variants are also described in the literature, which is of great importance for further research aimed at reducing the computation time and increasing the accuracy of the solutions obtained.

We also note that this model comprehensively covers a fairly large list of enterprise problems, which makes it possible to find solutions that are closer to the true optimal.

There are several points to the negative aspects of the model. It is difficult to speak about the rate of convergence of this method for large samples and large dimension of the problem. Probably, for larger samples or for larger problem dimensions, you will need to use numerical or heuristic algorithms.

The high requirements for the computer, together with the computational complexity of the problem, makes the process of debugging programs nontrivial in the case of a weak computer base and significantly increases the barrier to the practical implementation of the research results.

Based on the limitations and positive aspects of the research results, we will consider the directions for improving the model and the method for finding solutions.

At Step 1 of the two-stage optimization, it makes sense to use robust mixed-integer linear programming [24] due to the stochasticity of many variables, which should make it possible to speed up the search for a solution. The idea of this method is to identify constraints common to all iterations (deterministic constraints) and, in combination with some transformations of all remaining linear stochastic constraints, find a solution to the problem in a small number of iterations. This will significantly reduce the time to find a solution and the amount of RAM used.

Our assumptions about which goods should in the future be included in this or that volume in the production plan (based on Figure 2) must be further tested using the solution search scheme we developed.

It is necessary to replace the process of playing random variables with differential or other types of expressions, since otherwise a large amount of computation will be required, which in turn will affect the generation time of constraint matrices and objective functions for each iteration. After such a replacement, it will be necessary to use either heuristics or numerical methods. It is difficult to say how this type of replacement will affect problems of large dimension; additional research is needed.

It makes sense to connect other types of production processes to the model for assessing the feasibility of interaction between an enterprise and a timber commodity exchange in order to obtain more accurate results.

It should be noted that the transportation of finished products and raw materials by rail in reality is not deterministic. This is especially pro- 
nounced as the winter season approaches, when throughput decreases due to increased demand for the transport of other goods and types of raw materials (for example, fuel). In the future, it makes sense to add random variables describing the distance traveled over a period to obtain a more accurate solution. However, consideration of additional transportation factors will entail an increase in the calculation time and uncertainty in the algorithm for finding solutions.

It seems possible to use this model for planning production for the future, but in this case, it is necessary to think about the high-quality forecast of the situation on the market or on the commodity exchange. To obtain more accurate solutions, it also makes sense to consider the possibility of using deep learning of neural networks (or reinforcement learning) at the stage of purchasing raw materials, depending on how the situation in the raw materials market will develop.

\section{Conclusion}

This paper presents a model for solving the problem of forming sustainable supply chains and pricing policy of a timber industry enterprise. The model allows you to maximize the value of pre-tax profit and is a mathematical programming problem characterized by the complex possibility of simultaneously reckoning the production rates of consumption of raw materials for the production of final products, the formation of a vector of purchases of raw materials on a commodity exchange, the formation of a vector of volumes of transportation of finished products along the transport graph and the formation of an enterprise pricing policy. The results of the implementation of the model include the structure of production, the sequence of purchasing raw materials by production on the commodity exchange, the sequence of transporting the final product to buyers, as well as the value of profit for each day of operation of the enterprise and the price vector in the planning horizon under consid- eration. The process of finding a suboptimal solution to a problem is complicated by its large dimension, integer constraints and a rapidly growing load on RAM.

To solve such a problem, a two-stage optimization scheme was developed, consisting of steps with linear optimization and gradient descent. The work implements the two-stage Gomori method at the first step of the twostage optimization. From a practical point of view, the model is a tool that allows you to form a suboptimal production plan based on production factors and the volume of raw materials supply on the commodity exchange, as well as a plan for transporting the final product to buyers over the entire planning horizon. In the absence of the possibility of raising capital, the model allows you to find a suboptimal solution that allows an enterprise to avoid a cash gap.

The approbation of the model was carried out on the example of a timber processing complex from the Primorsky Territory. On the basis of the calculations performed and the solution found, recommendations were formulated for the management of the company in cooperation with the Russian Commodity Exchange. Analysis of the decision showed that despite the territorial proximity of the Irkutsk region to the Primorsky Territory, it is worth paying attention to the purchase of raw materials from the Moscow region and the Republic of Udmurtia. This is due to two reasons: sufficient potential in terms of extracted raw materials and a more acceptable pricing policy of forestry enterprises. A brief analysis of the possible production volumes of products of each type is carried out. It follows from the analysis that the production of most types of goods is not feasible. In general, the calculations allow us to make a conclusion about the possibility of rational procurement of raw materials on the commodity and raw materials exchange of Russia. 
To analyze qualitatively more complex business processes of an enterprise, it seems appropriate to modify the model by introducing probabilistic economic and production factors. However, in this case the question of a method for solving a complicated problem remains open. It also makes sense to consider the possibilities of speeding up the search for solutions.

\section{Acknowledgments}

The author expresses gratitude to the Educational Center NTU "Sirius" for the opportunity to use their computer base for testing the model, as well as to associate professor of the Moscow Institute of Physics and Technology (MIPT), professor of the Higher School of Economics, Dr. Alexander V. Gasnikov for valuable recommendations in the field of numerical optimization.

\section{References}

1. Pessôa M.V.P., Becker J.M.J. (2020) Smart design engineering: a literature review of the impact of the 4th industrial revolution on product design and development. Research in Engineering Design, no 31, pp. 175-195. DOI: 10.1007/s00163-020-00330-z.

2. Park S.H., Shin W.S., Park Y.H., Lee Y. (2017) Building a new culture for quality management in the era of the Fourth Industrial Revolution. Total Quality Management \& Business Excellence, vol. 28, no 9-10, pp. 934-945. DOI: $10.1080 / 14783363.2017 .1310703$.

3. Movahedipour M., Yang M., Zeng J., Wu X., Salam S. (2016) Optimization in supply chain management, the current state and future directions: A systematic review and bibliometric analysis. Journal of Industrial Engineering and Management, vol. 9, no 4, pp. 933-963. DOI: 10.3926/jiem.2035.

4. Chen D., Liu Z., Luo Z., Webber M., Chen J. (2016). Bibliometric and visualized analysis of energy research. Ecological Engineering, vol. 90, pp. 285-293. DOI: 10.1016/j.ecoleng.2016.01.026.

5. Fahimnia B., Sarkis J., Davarzani H. (2015) Green supply chain management: A review and bibliometric analysis. International Journal of Production Economics, vol. 162, no 101-114. DOI: 10.1016/j.ijpe.2015.01.003.

6. Accorsi R., Cascini A., Cholette S., Manzini R., Mora C. (2014) Economic and environmental assessment of reusable plastic containers: A food catering supply chain case study. International Journal of Production Economics, vol. 152, pp. 88-101. DOI: 10.1016/j.ijpe.2013.12.014.

7. Nooraie S.V., Parast M.M. (2016) Mitigating supply chain disruptions through the assessment of trade-offs among risks, costs and investments in capabilities. International Journal of Production Economics, vol. 171, part 1, pp. 8-21. DOI: 10.1016/j.ijpe.2015.10.018.

8. Liu Z., Hu B., Zhao Y., Lang L., Guo H., Florence K., Zhang S. (2020) Research on intelligent decision of low carbon supply chain based on carbon tax constraints in human-driven edge computing. IEEE Access, vol. 8 , pp. 48264-48273. DOI: 10.1109/ACCESS.2020.2978911.

9. Rogulin R.S., Mazelis L.S. (2020) Algorithm and mathematical model of supply chain management for raw wood from the regions in Russia: comparison and analysis. Perm University Herald. Economy, vol. 15, no 3, pp. 385-404 (in Russian). DOI: 10.17072/1994-9960-2020-3-385-404.

10. Tellez O., Daguet L., Lehuédé F., Monteiro T., Montoya G.O., Péton O., Vercraene S. (2020) A stakeholder oriented approach to the optimization of transports of people with disabilities. Supply Chain Forum: An International Journal, vol. 21, no 2, pp. 93-102. DOI: 10.1080/16258312.2020.1768435.

11. Scavarda L.F., Reichhart A., Hamacher S., Holweg M. (2010) Managing product variety in emerging markets. International Journal of Operations and Production Management, vol. 30, no 2, pp. 205-224.

12. Billal M., Hossain M. (2020) Multi-objective optimization for multi-product multi-period four echelon supply chain problems under uncertainty. Journal of Optimization in Industrial Engineering, vol. 13, no 1, pp. 1-17. DOI: 10.22094/JOIE.2018.555578.1529.

13. Ren J., Tan S., Yang L., Goodsite M.E., Pang C., Dong L. (2015) Optimization of emergy sustainability index for bio diesel supply network design. Energy Conversion and Management, vol. 92, pp. 312-321. DOI: 10.1016/j.enconman.2014.12.066.

14. Cardona-Valdés Y., Alvarez A., Ozdemir D. (2011) A bi-objective supply chain design problem with uncertainty. Transportation Research. Part C: Emerging Technologies, vol. 19, no 5, pp. 821-832. DOI: 10.1016/j.trc.2010.04.003.

15. El-Sayed M., Afia N., El-Kharbotly A. (2010) A stochastic model for forward-reverse logistics network design under risk. Computer \& Industrial Engineering, vol. 58, pp. 423-431. DOI: 10.1016/j.cie.2008.09.040. 
16. Schutz P., Tomasgard A., Ahmed S. (2009) Supply chain design under uncertainty using sample average approximation and dual decomposition. European Journal of Operational Research, vol. 199, no 2, pp. 409-419.

17. Chen C.-L., Lee W.C. (2004) Multi-objective optimization of multi-echelon supply chain networks with uncertain product demands and prices. Computers and Chemical Engineering, vol. 28, no 6-7, pp. 1131-1144. DOI: 10.1016/j.compchemeng.2003.09.014.

18. Georgiadis M.C., Tsiakis P., Longinidis P., Sofioglou M.K. (2011) Optimal design of supply chain networks under uncertain transient demand variations. Omega, vol. 39, no 3, pp. 254-272.

19. Wang K.-J., Makond B., Liu S.-Y. (2011) Location and allocation decisions in a two-echelon supply chain with stochastic demand - A genetic-algorithm based solution. Expert Systems with Application, vol. 38, no 5, pp. 6125-6131. DOI: 10.1016/j.eswa.2010.11.008.

20. Olivares-Benitez E., González-Velarde J.L., Ríos-Mercado R.Z. (2012) A supply chain design problem with facility location and bi-objective transportation choices. TOP, no 20, pp. 729-753. DOI: 10.1007/s11750-010-0162-8.

21. Rogulin R.S. (2020) A model for optimizing plans for procurement of raw materials from regions of Russia in a timber-processing enterprise. Business Informatics, vol. 14, no 4, pp. 19-35. DOI: 10.17323/2587-814X.2020.4.19.35.

22. Kan Yu.S., Kibzun A.I. (2009) Stochastic programming problems with probabilistic criteria. Moscow: FIZMATLIT (in Russian).

23. Kibzun A.I., Lebedev A.A., Malyshev V.V. (1984) On reducing a problem with probabilistic constraints to an equivalent minimax. Proceedings of the USSR Academy of Sciences. Engineering Cybernetics, no 4, pp. 73-80 (in Russian).

24. Kibzun A.I., Malyshev V.V. (1984) Generalized minimax approach to solving problems with probabilistic constraints. Proceedings of the USSR Academy of Sciences. Engineering Cybernetics, no 1, pp. 20-29 (in Russian).

25. Fedorov V.V. (1979) Numerical methods of maximin. Moscow: Nauka (in Russian).

26. Kanaeva O.N. (2004) A two-stage nonlinear stochastic programming problem with a deterministic compensation matrix. Mathematical Structures and Modeling, no 14, pp. 25-33 (in Russian).

27. Ozturk M.M., Cankaya I.A., Ipekci D. (2020) Optimizing echo state network through a novel Fisher maximization based stochastic gradient descent. Neurocomputing, vol. 415, pp. 215-224. DOI: 10.1016/j.neucom.2020.07.034.

28. Kennedy R.K.L., Khoshgoftaar T.M., Villanustre F., Humphrey T. (2019) A parallel and distributed stochastic gradient descent implementation using commodity clusters. Journal of Big Data, vol. 6, article no 16. DOI: 10.1186/s40537-019-0179-2.

29. Hassan M., Baharum A., Ali M.K.M. (2020) Logarithmic penalty function method for invex multi-objective fractional programming problems. Journal of Taibah University for Science, vol. 14, no 1, pp. 211-216, DOI: $10.1080 / 16583655.2020 .1715051$.

30. Price C.J. (2020) Direct search nonsmooth constrained optimization via rounded $1_{1}$ penalty functions. Optimization Methods and Software. DOI: 10.1080/10556788.2020.1746961.

31. Huang S., Li G., Ben-Awuah E., Afum B.O., Hu N. (2020) A robust mixed integer linear programming framework for underground cut-and-fill mining production scheduling. International Journal of Mining, Reclamation and Environment, vol. 34, no 6, pp. 397-414. DOI: 10.1080/17480930.2019.1576576.

\section{About the author}

\section{Rodion S. Rogulin}

Doctoral Student, Department of Mathematics and Modeling, Vladivostok State University of Economics and Service, 41, Gogolya Street, Vladivostok 690014, Russia;

Assistant Professor, Department of Applied Mathematics, Mechanics, Control and Software, Far Eastern Federal University, 10, Ajax Bay, Russky Island, Vladivostok 690922, Russia;

E-mail: rafassiaofusa@mail.ru

ORCID: 0000-0002-3235-6429 